\title{
Gout, not induced by diuretics? A case-control study from primary care
}

\author{
H J E M Janssens, E H van de Lisdonk, M Janssen, H J M van den Hoogen, \\ A L M Verbeek
}

See end of article for authors' affiliations .....................

Correspondence to: Dr H J E M Janssens, Department of General Practice and Family Medicine, 229 University Medical Centre, PO Box $9191,6500 \mathrm{HB}$, Nijmegen, The Netherlands; h.janssens@ hag.umen.nl

Accepted 4December 2005 Published Online First 16 November 2005
Background: It is taken for granted that diuretics may induce gout, but there is a general lack of evidence on this topic.

Objectives: To determine the incidence of gout in patients who use diuretics, taking into account concurrent hypertension and cardiovascular diseases.

Methods: A case-control study was designed. From a primary care population all patients with a first gout registration (59 men, 11 women; mean (SD) age 55.1 (13.5)) were identified as cases. To relate the occurrence of gout to diuretic use a matched reference series of three controls for each case was compiled. Conditional logistic regression analyses were applied to estimate incidence rate ratios (IRRs) of gout, and 95\% confidence intervals (Cls), in subjects with and without diuretic treatment, hypertension, and cardiovasculardiseases. Additional stratification analyses were made, particularly in the subjects not using diuretics.

Results: The IRRs of gout in subjects with $v$ those without diuretic treatment, hypertension, heart failure, and myocardial infarction were $2.8(95 \% \mathrm{Cl} 1.2$ to 6.6$), 2.6(95 \% \mathrm{Cl} 1.2$ to 5.6$), 20.9(95 \% \mathrm{Cl} 2.5$ to 173.8$)$, and $1.9(95 \% \mathrm{Cl} 0.7$ to 4.7$)$, respectively. After adjustment, the IRR of gout for diuretic use dropped to 0.6 (95\% $\mathrm{Cl} 0.2$ to 2.0 ), while the IRRs of gout for hypertension, heart failure, and myocardial infarction were still $>1$. This was also the case for subjects with hypertension or myocardial infarction, who had not used diuretics.

Conclusion: The results suggest that diuretics do not actually increase the risk of gout. Cardiovascular indications for treatment may have confounded previous inferences.
D iuretics are drugs with a widespread use, with a spectrum of evidence based, important indications (hypertension, cardiovascular diseases). They are safe, cheap, and have minimal side effects. Of these side effects, the possibility of the induction of gout has been debated for decades. ${ }^{12}$ Diuretics are supposed to have a direct effect on ion exchanger proteins at the proximal tubule lumen membrane in the kidney which enhance the urate reabsorption, resulting in higher blood levels of uric acid. ${ }^{2}$ These higher levels reach a supersaturation level, and this is followed by shedding and precipitation of urate crystals into joints or subcutaneous tissues (tophi). ${ }^{3}$ The joint precipitation activates cellular signal transducers and induces inflammatory mediators, resulting in gouty synovitis. ${ }^{23}$ Although we found a general lack of reported evidence about this whole process of gout induction, gout as a side effect of diuretics has been generally accepted, and this has had an effect on medical practice. In the guidelines for the treatment of hypertension, for example, gout has been formulated as a compelling contraindication to the prescription of diuretics. ${ }^{45}$ Many people consider that the increasing number of diuretic treatments (prescriptions and defined daily dosages) is one of the main contributory factors to the increased prevalence and incidence of gout in Western industrialised countries. ${ }^{26}$

The indications to prescribe diuretics (hypertension and cardiovascular diseases) are themselves also associated with gout, as has been showed by several epidemiological studies. ${ }^{7-9}$ Other studies have concluded that patients with hypertension and cardiovascular diseases have increased xanthine oxidase activity and have higher uric acid serum levels. ${ }^{10-15}$ Thus, a pathogenic basis for understanding the associations of gout, hypertension, and cardiovascular morbidity becomes apparent, apart from the use of diuretics. $^{216}$

We found disputable reported results in the studies on the side effects of diuretics, including the incidence of gouty arthritis. ${ }^{17-24}$ We concluded that these studies did not take into account the possible confounding by the indications to prescribe diuretics. Also knowledge that the number of diuretic prescriptions is increasing was sufficient reason to design a case-control study on this topic. We performed this study in a primary care setting formulating the research question: is there a higher incidence of gout in patients using diuretics than in non-users, which is independent of treatment indications (hypertension, cardiovascular morbidity)?

\section{METHODS}

In our study we used the records of patients enlisted in October 2002, in a Dutch primary healthcare centre. This centre, located in a rural area in the east of the Netherlands, provided total pharmaceutical care for all its enlisted patients. Most of them were white, and comparable to the general Dutch population for age, sex, and social class. As neither the use of diuretics nor gout is prevalent below the age of 35 years, we restricted our source population to people aged 35 and older in October 2002. During the defined study period October 1994 to October 2002 the size of this population was almost constant. Several chronic diseases (among others hypertension, diabetes mellitus, asthma, emphysema) were coded according the International Classification of Health Problems in Primary Care (ICHPPC-2)..$^{25}$ Cardiovascular morbidity was recorded by a code that comprises heart failure, myocardial infarction, angina pectoris, cerebrovascular accident, transient ischaemic attack, and peripheral 
vascular disease. Drugs dispensed in the pharmacy had electronically accessible codes according to the Anatomical Therapeutic Chemical system of drug classification created by the World Health Organisation. ${ }^{26}$

Using the data from the source population, we performed a case-control study to examine the possibility of an association of gout with diuretic use. We identified as cases all patients with a first gout registration. The gout diagnoses of all cases were reassessed retrospectively by investigating their medical reports. We accepted the diagnosis of gout if there was documentation of urate crystals aspirated from an affected joint, or, failing this, if the clinical characteristics recorded were sufficient to fulfil the diagnostic criteria of gout as formulated by the American Rheumatology Association. ${ }^{27}$ We excluded patients with a first case of gout before the study period, after checking their reports before October 1994. We sampled three random controls for each case. These were people from the source population of the same age and sex, but who did not have gout. The cases and controls were characterised by diuretic use, hypertension, all cardiovascular morbidity, and specific cardiovascular diseases. Diuretic use was defined as a dispensation in the pharmacy of diuretic tablets (ATC-code C03), with a prescription that should have resulted in the daily therapeutic use during a minimal period of three consecutive months. The diagnoses of hypertension, and the specific cardiovascular diseases (heart failure, myocardial infarction, angina pectoris, cerebrovascular accident, transient ischaemic attack, and peripheral vascular disease) were retrospectively evaluated, and only accepted, if diagnostic criteria from the guidelines of the Dutch College of General Practitioners were fulfilled. ${ }^{28}$

Using conditional logistic models, we first performed univariate analyses to calculate the incidence rate ratio (IRR) of gout, and 95\% confidence intervals (CIs), for subjects with the variables diuretic use, hypertension, and cardiovascular diseases with indication for diuretic treatment (heart failure and myocardial infarction), compared with subjects without the variable. Thereafter, IRRs were calculated multivariately, taking into account hypertension, heart failure, and myocardial infarction.

As colinearity between the studied variables with diuretic use could be expected, we carried out additional univariate analyses with stratification based on diuretic use. We estimated the IRR of gout, comparing subjects with and without the variable. To perform the analyses we used the SAS-System software (version 8.2; 1999-2001).

\section{RESULTS}

The source population comprised 3764 people aged 35 and older (men 1913). The prevalence of hypertension and all cardiovascular morbidity was, respectively, $11.1 \%$ and $9.0 \%$.

Table 1 Characteristics of cases and controls

\begin{tabular}{|c|c|c|c|}
\hline & $\begin{array}{l}\text { Cases } \\
(n=70)\end{array}$ & $\begin{array}{l}\text { Controls } \\
(n=210)\end{array}$ & p Value \\
\hline Men/women & $59 / 11$ & $177 / 33$ & \\
\hline Age (years), mean (SD) & $55.1(13.5)$ & $55.2(13.5)$ & \\
\hline Diuretic use* & $14(20)$ & $20(10)$ & 0.020 \\
\hline Hypertension & $15(21)$ & $21(10)$ & 0.013 \\
\hline All cardiovascular & & & \\
\hline morbidity $†$ & $17(24)$ & $22(10)$ & 0.004 \\
\hline Heart failure & $7(10)$ & $2(1)$ & 0.000 \\
\hline Myocardial infarction & $8(11)$ & $14(7)$ & 0.200 \\
\hline
\end{tabular}

Results shown as absolute numbers (\%) unless stated otherwise. *Daily use during a minimal period of 3 consecutive months; tcomprising heart failure, myocardial infarction, angina pectoris, cerebrovascular accident, transient ischaemic attack, and peripheral vascular disease.
Table 2 Incidence rate ratios (IRRs) of gout during diuretic use, and during diseases with indications for diuretic treatment; results after univariate and multivariate conditional analyses

\begin{tabular}{lll}
\hline & IRR of gout $(95 \% \mathrm{Cl})$ \\
\cline { 2 - 3 } & Univariate analysis & Multivariate analysis \\
\hline Diuretic use & $2.8(1.2$ to 6.6$)$ & $0.6(0.2$ to 2.0$)$ \\
Hypertension & $2.6(1.2$ to 5.6$)$ & $3.9(1.6$ to 10.0$)$ \\
Heart failure & $20.9(2.5$ to 173.8$)$ & $40.1(3.8$ to 437.2$)$ \\
Myocardial infarction & $1.9(0.7$ to 4.7$)$ & $1.5(0.5$ to 4.1$)$ \\
\hline
\end{tabular}

During the study period $14.0 \%$ of the source population had ever received one or more diuretics (incidence). Knowing that the number of enlisted patients was almost constant during the study period we estimated an overall incidence rate of gout: 232 per 100000 person years (men 386). Patients, who had ever received a diuretic, had a relative risk of gout estimated at 1.56. For patients with known hypertension, and cardiovascular morbidity the relative risks of gout were 2.18 and 3.26, respectively. Table 1 gives details of the characteristics of the cases and controls. Diuretic use, hypertension, and cardiovascular morbidity occurred about twice as much in cases as in controls. The duration of diuretic use varied from 3 to 93 months.

Table 2 shows the IRR of gout after univariate and multivariate analyses, comparing subjects with and without diuretic use, hypertension, heart failure, and myocardial infarction. The IRR of gout, estimated univariately, reached statistical significance for diuretic use, hypertension, and heart failure. The results of the multivariate conditional logistic regression, adjusted for diuretic use, prevalent hypertension, prevalent heart failure, and myocardial infarction showed statistical significance for hypertension and heart failure. For diuretic use the IRR of gout was 0.6 (95\% CI 0.2 to 2.0 ). After stratification based on diuretic use the IRR of gout in the subgroup of non-users with hypertension was $4.2(95 \%$ CI 1.5 to $11.6 ; \mathrm{p}=0.005)$ compared with non-users without hypertension. For myocardial infarction in comparison with no infarction this IRR was 2.5 (95\% CI 0.8 to 7.5 ; $\mathrm{p}=0.112$ ).

\section{DISCUSSION}

In the source population we found that a considerable percentage of patients had ever used a diuretic-namely, $14 \%$. This is in agreement with the increasing use of diuretics, as noted by others. ${ }^{2}{ }^{6}$ The estimated relative risk for patients from the source population who ever used a diuretic was 1.56, suggesting no important risk of gout (number needed to harm: 107). Relative risks of getting gout for patients with hypertension or cardiovascular morbidity were highernamely, 2.18 and 3.26, respectively.

In our case-control study there was a higher IRR of gout when subjects used diuretics for at least three consecutive months: 2.8 (95\% 1.2 to 6.6). However, after correction for the indications for treatment this IRR was 0.6 (95\% CI 0.2 to 2.0 ), indicating no statistical support for a real increased risk of gout that is independent of these indications. Higher IRRs of gout independent of diuretics (after stratification) were seen in patients with hypertension compared with no hypertension (statistically significant), and in patients with myocardial infarction compared with no infarction (not statistically significant). The high IRR for heart failure after univariate and multivariate analyses was remarkable. Caution is required in interpreting this, as the 95\% CI is wide, and because statistical interaction with diuretic use 
could not be studied separately, as all patients with heart failure used diuretics, in accordance with normal medical practice. However, in published reports we found strong arguments for metabolic conditions associated both with gout and with heart failure, which were independent of the use of diuretics. ${ }^{12-14}$

Morbidity (gout, hypertension, cardiovascular morbidity) and the use of drugs (diuretics) were examined simultaneously in the present study. The validity of our data was probably enhanced by the use of electronic data from a single medical centre, which supplied both the primary health care and the drugs to all the participants. Others have confirmed the reliability of research data from drug dispensing general practitioners in the Netherlands. ${ }^{29}$

The case-control study design has been proved to be a suitable process for investigating the risks of side effects of drugs, as we did in the present study. ${ }^{30}$ We were aware of the methodological problems of the design: information bias, selection bias, and confounding by indication. These methodological points probably do not interfere seriously with the results of our study, as we discuss here.

Morbidity data were already registered before we started our study. By rediagnosing this morbidity independently from drug data, and according to diagnostic guidelines, diagnostic misclassification of gout, and misclassification of hypertension, heart failure, and myocardial infarction were excluded as much as possible. If misclassification still exists it should apply equally to cases and controls, with a certain risk of underestimation.

The overall incidence of gout per 100000 person years in our primary care source population was compatible with other epidemiological results reported in Dutch primary care. $^{31}{ }^{32}$ Knowing that almost all first gout attacks will present to a doctor, the gout incidence in Dutch primary care probably corresponds well with the incidence in the general population. A sudden red, swollen, and very painful joint urges a patient to seek medical help, in particular when it is the first attack, even during the weekend. In the Dutch healthcare system the general practitioners are in such a case the first, and often the only, doctors who will take care of these incidents.

We used pharmacy dispensation data of diuretics to measure the use of the drugs by study participants. This may be a limitation, but we think our data should have accurately reflected real use, as almost all dispensations were based on direct requests by patients to their doctor to repeat a prescription when their tablets had run out. Hence, we think there was hardly any risk of misclassification of the level of diuretic use.

A literature search failed to disclose any information about the minimal use (dosage and duration) of diuretics which might possibly elicit an attack of gout. So we made an arbitrary choice: a daily dose of at least one diuretic for at least 3 months. In the study we did not include the exact dosage and duration of use, but we noticed that all cases and controls receiving the drugs had used a therapeutic dosage. The duration of use in three subjects (one case and two controls) was between 3 and 6 months and in the rest between 11 and 93 months.

Following the contraindications for the major classes of antihypertensive drugs that have been described in the international guidelines on hypertension, ${ }^{45}$ no distinction was made between loop diuretics and thiazide diuretics in this study.

Cases and controls had the same chance to become a participant in the study, and they had the same chance of receiving diuretics or having hypertension and cardiovascular morbidity. For reasons of homogeneity we studied incident cases-that is, patients who got gout for the first time during the study period. These conditions excluded selection bias as much as possible.

We were not able to quantify other possible confounding factors like obesity, alcohol use, and impaired renal function. Furthermore, the number of variables was small, which undermines the robustness of the statistical conclusions.

We found only two studies originally designed to study primarily the relationship between diuretics and gouty arthritis. ${ }^{17} 18$ These studies did not analyse for possible confounding cardiovascular factors, but concluded that diuretic-induced gout occurs particularly in elderly women, ${ }^{17}$ and in patients in whom there is an additional cause of hyperuricaemia, usually impaired renal function. ${ }^{18}$ Other studies on this topic did not adequately define gout and diuretic use, as we tried to do; or they had restricted entry criteria for age, sex, or blood level of uric acid of the participants, which we did not. ${ }^{20-24}$ We found no studies in a primary healthcare setting, or with a comparable design. Hence, we were not able to compare our results with those of other studies.

In our study there was hardly any evidence for "gout induced by diuretics". Hence, we think there is no reason to withhold diuretics from patients because of a risk of gout. Furthermore, there are no arguments for changing diuretics to drugs with a totally different working principle and profile (for example, $\beta$ blockers, angiotensin converting enzyme inhibitors or calcium channel blockers) when a patient gets gout, as diuretics have a good reputation, as has been reviewed..$^{33}$ We do not think gout should be considered as a compelling contraindication to prescription of a diuretic. Hypertension and cardiovascular morbidity are the factors which should be considered when a patient with gout visits his doctor, thus putting the importance of diuretics and gout into perspective.

\section{ACKNOWLEDGEMENTS}

We thank the drug dispensing general practitioners of the Health Centre Lobede, Lobith-Tolkamer, The Netherlands, for the use of their electronic data; and Hans Bor, statistician, (University Medical Centre, Nijmegen, The Netherlands) for helping us with the data analysis.

\section{Authors' affiliations}

H J E M Janssens, E H van de Lisdonk, H J M van den Hoogen, University Medical Centre, Department of General Practice and Family Medicine, Nijmegen, The Netherlands

M Janssen, Department of Rheumatology, Rijnstate Hospital, Arnhem, The Netherlands

A L M Verbeek, University Medical Centre, Department of Epidemiology and Biostatistics, Nijmegen, The Netherlands

Conflict of interest: None.

\section{REFERENCES}

1 Humphreys DM. Acute gout apparantly precipitated by furosemide. BMJ 1966;1:1024-5

2 Bieber JD, Terkeltaub RA. Gout: on the brink of novel therapeutic options for an ancient disease. Arthritis Rheum 2004;50:2400-14

3 Pascual E. Management of crystal arthritis. Reumatology (Oxford) 1999;38:912-6.

4 European Society of Hypertension. 2003 European Society of HypertensionEuropean Society of Cardiology guidelines for the management of arterial hypertension. J Hypertens 2003;21:1011-53.

5 World Health Organization. 1999 World Health Organization-International Society of Hypertension guidelines for the management of hypertension. $J$ Hypertens 1999;17:151-83.

6 College van Zorgverzekeringen. Geneesmiddelen Informatie Project. Available from, http://www. gipdatabank.nl/ (accessed 15 April 2006).

7 Abbott RD, Brand FN, Kannel WB, Castelli WP. Gout and coronary heart disease: the Framingham Study. J Clin Epidemiol 1988;41:237-42.

8 Hochberg MC, Thomas J, Thomas DJ, Mead L, Levine DM, Klag MJ. Racial differences in the incidence of gout. The role of hypertension. Arthritis Rheum 1995;38:628-32 
9 Janssens HJEM, van de Lisdonk EH, Bor $H$, van den Hoogen $H J$, Janssen $M$. Gout, just a nasty event or a cardiovascular signal? A study from primary care. Fam Pract 2003:20:413-16.

10 Johnson RJ, Kang DH, Feig D, Kivlighn S, Kanellis J, Watanabe S, et al. Is there a pathogenetic role for uric acid in hypertension and cardiovascular and renal disease? Hypertension 2003;41:1183-90.

11 Feig DI, Johnson RJ. Hyperuricemia in childhood primary hypertension. Hypertension 2003:42:247-52.

12 Leyva F, Anker S, Swan JW, Godsland IF, Wingrove CS, Chua TP, et al. Serum uric acid as an index of impaired oxidative metabolisme in chronic heart failure. Eur Heart J 1997; 18:858-65.

13 Anker SD, Doehner W, Rauchhaus M, Sharma R, Francis D, Knosalla C, et al. Uric acid and survival in chronic heart failure: validation and application in metabolic, functional, and hemodynamic staging. Circulation 2003;107:1991-7.

14 Hare JM, Johnson RJ. Uric acid predicts clinical outcomes in heart failure: insights regarding the role of xanthine oxidase and uric acid in disease pathophysiology. Circulation 2003;107:1951-3.

15 Wannamethee SG, Shaper AG, Whincup PH. Serum urate and the risk of major coronary heart disease events. Heart 1997:78:147-53.

16 Terkeltaub RA. Gout. N Engl J Med 2003;349:1647-55.

17 Macfarlane DG, Dieppe PA. Diuretic-induced gout in elderly women. Br J Rheumatol 1985;24:155-7.

18 Scott JT, Higgens CS. Diuretic induced gout: a multifactorial condition. Ann Rheum Dis 1992;51:259-61.

19 Berglund G, Andersson O, Widgren B. Low-dose antihypertensive treatment with a thiazide diuretic is not diabetogenic. A 10-year controlled trial with bendroflumethiazide. Acta Med Scand 1986;220:419-24.

20 Langford HG, Blaufox MD, Borhani NO, Curb JD, Molteni A, Schneider KA, et al. Is thiazide-produced uric acid elevation harmful? Analysis of data from the Hypertension Detection and Follow-up Program. Arch Intern Med 1987; 147:645-9.

21 Fletcher AE. Adverse treatment effects in the trial of the European Working Party on High Blood Pressure in the Elderly. Am J Med 1991;90(3A):42S-4S

22 Staessen J. The determinants and prognostic significance of serum uric acid in elderly patients of the European Working Party on High Blood Pressure in the Elderly trial. Am J Med 1991;90(3A):50S-4S
23 Gurwitz JH, Kalish SC, Bohn RL, Glynn RJ, Monane M, Mogun H, et al. Thiazide diuretics and the initiation of anti-gout therapy. J Clin Epidemiol 1997:50:953-9.

24 Lin KC, Lin Hy, Chou P. The interaction between uric acid level and other risk factors on the development of gout among asymptomatic hypeuricemic men in a prospective study. J Rheumatol 2000;27:1501-5.

25 Classification Committe of WONCA. ICHPPC-2 defined, 3rd ed. Oxford: Oxford University Press, 1983.

26 World Health Organisation. The Anatomical Therapeutic Chemical Classification System with Defined Daily Doses (ATC/DDD). Available from, http://www.who.int/classifications/atcddd/en/print.html (accessed 15 April 2006).

27 Wallace SL, Robbinson H, Masi AT, Decker JL, McArty DJ, Yü T. Preliminary criteria for the classification of the acute arthritis of primary gout. Artritis Rheum 1977;20:895-900.

28 Dutch College of General Practitioners. NHG-standaarden Hypertensie, Hartfalen, Coronair Syndroom, Angina pectoris, TIA, CVA en PAV. Available from, http://nhg.artsennet.nl/content/resources/ AMGATE_6059_104_TICH_L748581710/ AMGATE_6059_104_TICH_R1 19952487066081// (accessed 15 April 2006).

29 Ottervanger JP, van Witsen TB, Valkenburg HA, Grobbee DE, Stricker BH. Adverse reactions attributed to sumatriptan. A postmarketing study in general practice. Eur J Clin Pharmacol 1994;47:305-9.

30 Miettinen OS. Estimability and estimation in case-referent studies. Am J Epidemiol 1976;103:226-35.

31 Lisdonk EH, Van den Bosch WJHM, Huygen FJA, Lagro-Jansen ALM. Ziekten in de huisartspraktiik [Diseases in general practice]. Maarssen: Elsevier, 2003

32 Gorter KJ, Tan G, Verstappen WHJM, Cirkel JW, Kolnaar BGM, Osterberg E, et al. NHG-standaard Jicht [gout]. Available from, http://nhg.artsennet.nl/ upload/104/guidelines2/E72.htm\#noot4 (accessed 15 April 2006)

33 Psaty BM, Lumley T, Furberg CD, Schellenbaum G, Pahor M, Alderman MH, et al. Health outcomes associated with various antihypertensive therapies used as first-line agents. A network meta-analysis. JAMA 2003;289:2534-44.

34 Faris R, Flather $M$, Purcell $H$, Henein $M$, Poole-Wilson P, Coats A. Current evidence supporting the role of diuretics in heart failure: a meta-analysis of randomised controlled trials. Int J Cardiol 2002;82:149-58. 